\title{
Integrative therapies in cancer patients: a look at the experience in a university extension group in Brazil
}

Keywords: cancer, integrative therapies, acupuncture, relaxation, quality of life

Abbreviations: WHO, world health organization; QVRS, health related quality of life; PNPIC, national policy on integrative and complementary practices

\section{Introduction}

The number of cancer cases has increased significantly each year. According to the World Health Organization (WHO), a drastic increase is expected over the coming decades and may account for a population impact of $80 \%$ from more than 20 million new cases by $2025 .^{1}$

It is known that cancer and its treatment causes several symptoms and harmful side effects to patients, which negatively compromises the Health-Related Quality of Life (HRQoL) during treatment, and may also interfere with adherence to cancer treatment.

In this context, an extension group was created at a University of the Interior of the State of São Paulo, since 1990, composed of nurses, physician and physiotherapist, who develop activities with cancer patients undergoing chemotherapy, in order to alleviate the resulting symptoms of the treatment and provide improvement of the HRQoL of these patients during treatment.

The research group uses the Integrative Therapies of Acupuncture and Relaxation with Guided Visualization as strategies to relieve symptoms of nausea, vomiting, pain, anxiety, fatigue, among others. The group meetings with the patients take place every week, every Monday, where the Integrative Therapies sessions are held.

In Brazil there is the National Policy on Integrative and Complementary Practices (PNPIC), with the objective of providing the population with the offer of Integrative Practices within the Unified Health System (SUS), ${ }^{2}$ which consists of a public health system of free access to any Brazilian citizen, seeking to offer integral attention to the health of its users.

Within this perspective of integral health care, Integrative Therapies are employed in order to provide a holistic care model, balancing the technology of conventional therapies with humanization in the care and use of simpler, less costly resources that ameliorate the side effects of conventional treatment, such as chemotherapy. ${ }^{3}$

Acupuncture is an ancient practice developed by Chinese Traditional Medicine and the diagnosis of this therapy is based on the identification of energy imbalances, seeking to reestablish the individual's organic, emotional and mental balance. ${ }^{2}$ This therapy is well accepted by the patients participating in the extension group, who report immediate improvements in the reported symptoms.

Guided Visualization Relaxation is a mind-body technique, which also seeks organic balance through deep breathing, relaxation of the body, and also of the mind through guided visualization. The mind-

\author{
Volume 2 Issue 6 - 2018
}

\author{
Juliana Maria de Paula,' Amanda Fonseca \\ Baviera,' Bruna Francielle Toneti,' Rafael \\ Mendes Barbosa,' Liyoko Okino, ${ }^{2}$ Namie \\ Okino Sawada \\ 'Department of Nursing, Ribeirão Preto College of Nursing of \\ University of São Paulo, Brazil \\ ${ }^{2}$ Liyoko Clinic, Dermatology and Acupuncture, Brazil
}

Correspondence: Juliana Maria de Paula, University of São Paulo, Antônio Vasques, 374, Zip code 14095-080, Ribeirão Preto, SP, Brazil, Email jm_paula@hotmail.com

Received: October II, 2017 | Published: December 20, 2018

body model argues that the mental and emotional state has a direct link with the onset of diseases such as cancer, and, in the same way, also has an influence on the recovery and cure of the disease. ${ }^{4}$

In cases of advanced cancer, the prognosis is not always good and the patient often receives conventional treatment for palliative purposes and, thus, the use of integrative therapies together, provides comfort to the patient. The benefits of Integrative Therapies in patients in palliative care are related to the relaxation promoted by certain therapies, professional and patient interaction, potentiation of the effects of medications against pain and emotional well-being. ${ }^{5}$

The use of Integrative and Complementary Therapies has been gaining ground in the oncological context, since these therapies are easy to apply, low cost and safe to use. The beneficial effects of these therapies are easily noticed, such as reduction of pain, nausea, fatigue, in addition to physical recovery, there is the emotional restoration of the patient.

\section{Acknowledgments}

None.

\section{Conflicts of interest}

The authors declare there is no conflict of interest.

\section{References}

1. Brasil. National Cancer Institute José Alencar Gomes da Silva. Estimate 2016: Incidence of cancer in Brazil. Rio de Janeiro: INCA; 2017.

2. Brasil. Ministry of Health. Secretariat of Health Care. Department of Basic Attention. National Policy on Integrative and Complementary Practices in Health. Brasilia, 2013.

3. Borges MR, Madeira LM, Azevedo VMGO. Complementary and integrative practices in women's health care: a strategy for the humanization of medical care at Sofia Feldman Hospital. Rev Min Enferm. 2001;15(1):105-113. 
4. Simonton OC, Matthews-Simonton S, Creighton JL. With life again: a self-help approach for cancer patients. São Paulo: Summus Editorial; 1987:238.
5. Caires JS, Andrade TA, Amaral JB, et al. The use of complementary therapies in palliative care: benefits and purposes. Cogitare Enfermagem. 2014;19(3):515-520. 